\title{
Réaction individuelle de hêtres (Fagus silvatica L.) d'âges divers à diverses intensités d'éclaircie
}

\author{
J. Bouchon ', J.F. Dhôte ${ }^{1}$, et L. Lanier ${ }^{2}$ \\ 1 INRA, Centre de Recherches Forestières de Nancy, Champenoux, F 54280 Seichamps; \\ ${ }^{2}$ ENGREF, Centre de Nancy, 14, rue Girardet, F 54042 Nancy, France
}

(reçu le 26 septembre 1988, accepté le 17 octobre 1988)

\begin{abstract}
Résumé - Une expérience d'éclaircie sur 4 peuplements de hêtres d'âges étagés de 37 à 70 ans a montré que, même à un âge assez avancé, les houppiers réagissent rapidement et fortement aux éclaircies. Ce développement du houppier, accompagné secondairement d'un allongement de la saison de végétation, explique des gains importants en croissance radiale. Des éclaircies fortes et précoces sont donc recommandées pour le hêtre; la sélection d'arbres de place semble possible, même avant 40 ans; cependant, des inversions de rang social ayant été observées dans les peuplements les plus jeunes, des réajustements risquent d'être nécessaires lors de la seconde éclaircie. Enfin, tous ces résultats ayant été obtenus sur des placettes de faible surface, il resterait à les confirmer en peuplement.
\end{abstract}

croissance des arbres - éclaircie - âge - hêtre - Fagus silvatica L. - Lorraine

Summary - Different thinning levels at different ages : influence on individual growth of beeches (Fagus silvatica L.). A thinning experiment in 37 to 70 -year-old Beech (Fagus silvatica L.) stands shows that, even at late ages, crowns react quickly and vigorously to thinning. This crown development in turn, along with a secondary increase of the vegetation period, accounts for important gains in breast height diameter growth. Thus, it is recommanded that thinnings be heavy and early in Beech; the selection of crop trees seems possible, even before the age of 40; however, as social regressions have been observed in the youngest plots, reselection may prove to be necessary by the time of the second thinning. These results are based on observations on small plots : they should also be confirmed on larger stands.

tree growth - thinning - age - beech - Fagus silvatica L. - Lorraine

\section{Introduction}

La qualité recherchée pour les usages les plus rémunérateurs du bois de hêtre (déroulage, ébénisterie fine) dépend sensiblement du traitement sylvicole, tant en ce qui concerne les propriétés physiques (densité, dureté, rétractabilité) que mécaniques (contraintes de croissance) : les travaux de Poige (1973), Keller et al. (1976), Polge (1981), Ferrand (1982), qui portent sur des hêtraies du Nord de la France, représentant une large gamme de 
stations écologiques et de types de sylviculture, font ressortir que la qualité du bois produit par un arbre est d'autant meilleure que celui-ci occupe une position sociale plus élevée (en termes de dominance) et possède un houppier plus développé. L'arbre le plus désirable, dans ces conditions, est donc un arbre de type utaillissous-futaie".

Du point de vue de la sylviculture à appliquer aux peuplements de hêtre, ces résultats plaident pour la pratique d'éclaircies vigoureuses, visant au développement rapide des houppiers d'individus sélectionnés. A cette fin, la technique de désignation d'arbres d'avenir (sélection précoce d'arbres destinés à constituer la récolte finale et au profit desquels seront conduites les éclaircies) semble tout à fait adaptée.

$\mathrm{Si}$, pour résumer, on veut produire en futaie des arbres de type "taillis-sousfutaie", les questions suivantes se posent :

- quelle est la capacité des arbres à profiter d'une éclaircie pour reconstituer un large houppier ?

- cette capacité dépend-elle de l'âge, du traitement antérieur, de l'intensité des éclaircies?

- y a-t-il un risque à pratiquer des interventions trop énergiques ou trop précoces (pertes de production, inversions de rang social, descentes de cime) ?

- si c'est le cas, peut-on définir des intervalles (d'âge à la désignation, d'intensité d'éclaircie) optimaux pour l'objectif de qualité recherché et compte tenu des risques courus?

Le dispositif expérimental de la route de Frouard, en forêt de Haye (54), a été installé en 1976 pour répondre à ces ques- tions. Dans la conception de ce dispositif, l'accent a été mis sur la description précise, au niveau individuel,

- des caractéristiques dendrométriques classiques et du développement aérien de l'arbre;

- d'aspects qualitatifs et morphologiques : état sanitaire, forme, défauts, gourmands...

Ce sont principalement les résultats concernant le premier aspect (croissance du fût et développement du houppier) qui seront présentés ici.

\section{Matériel et Méthodes}

\section{Matériel}

Dans une zone assez homogène, quant à la station, au relief et à l'exposition, où le hêtre est très largement dominant, un certain nombre de parcelles ont été choisies, dans des peuplements d'âges variés : il s'agit des parcelles 67 , $68,36,117,110$ et 113 de la forêt de Haye.

Le dispositif est constitué de 320 placettes monoarbres réparties en 8 blocs de 40 arbres: deux blocs de 37 ans, un de 48 , trois de 58 et deux de 70 ans à la fin de 1976. Ces âges moyens ont été déterminés a posteriori, après la première éclaircie, exploitée en 1976. Cinq traitements ont été appliqués, correspondant à 5 niveaux d'éclaircie croissante : ils sont décrits plus en détail plus bas, p. 254.

Les 320 arbres ont été choisis en fonction des critères (potentiel de croissance et morphologie) habituellement utilisés par les sylviculteurs pour désigner des arbres d'avenir :

- arbres dominants (quelques codominants ont intentionnellement été conservés dans l'étude),

- houppier équilibré,

- tige suffisamment rectiligne, verticale, ayant une forme convenable et un élagage correct.

Autour de chacun des 320 arbres retenus, une placette circulaire de 1 are $(0,0247$ acre) a été délimitée, ainsi qu'une couronne circulaire de 1 are supplémentaire. 
Mesures et notations

Les mesures suivantes ont été effectuées sur l'arbre central choisi :

- surface terrière à $1,30 \mathrm{~m}$, hauteur totale de l'arbre, codage de la position sociale;

- description du houppier : projection horizontale, hauteur de la base, de la plus large extension;

- mesure de la rectitude, notations des défauts (branches mortes, gourmands), des attaques cryptogamiques ou parasitaires.

Ces mesures ont été faites à chaque passage en éclaircie (rotation de 5 ans) depuis l'installation de l'expérience en 1976. De plus, des rubans microdendrométriques ont été posés sur 60 arbres pour étudier si l'éclaircie avait un effet sur les rythmes annuels de croissance.

Dans la suite du texte, les symboles $h, g$, et $k$ seront utilisés pour représenter respectivement la hauteur, la surface terrière et la surface de la projection horizontale du houppier. L'année de référence sera 1976 et les indices correspondront aux années d'observation : ainsi, g81 sera la surface terrière en 1981.

On emploiera I pour désigner des accroissements et IR pour les accroissement relatifs. Ainsi,

$$
\operatorname{IR} h_{81}=\frac{h_{81}-h 76}{h_{76}}
$$

sera l'accroissement en hauteur de 1976 à 1981, rapporté à la hauteur initiale $h_{76}$.
Tableau I. Regroupement des 8 blocs initiaux de 40 arbres en 4 blocs homogènes de 80 arbres.

\begin{tabular}{lll}
\hline Parcelle(s) & $\begin{array}{l}\text { Age moyen } \\
\text { fin 1976 }\end{array}$ & $\begin{array}{l}\text { Nombre } \\
\text { d'arbres }\end{array}$ \\
\hline 113 & 70 ans \pm 16 ans & 80 \\
110 & 58 ans \pm 9 ans & 80 \\
36 et 117 & 53 ans \pm 15 ans & 80 \\
67 et 68 & 37 ans \pm 9 ans & 80 \\
\hline
\end{tabular}

Etat initial du peuplement et recomposition du dispositif

On a cherché à caractériser l'état initial des peuplements avant la première éclaircie (1976) en considérant, pour les 320 arbres, les 3 variables $g_{76}, h_{76}$ et $k_{76}$. Une analyse discriminante a été effectuée sur les 8 groupes de 40 arbres, décrits par ces 3 variables; si l'on considère les distances entre ces groupes (il s'agit des distances de Mahalanobis (Dagnelie, 1975, pp 229-236), il apparaît qu'on peut regrouper 2 à 2 ces groupes en 4 blocs homogènes de 80 arbres (Tableaux I et II). Signalons notamment qu'un des groupes de 58 ans est très voisin du lot de 48 ans, c'est-à-dire que les hauteurs, surfaces terrières et surfaces de projection de houppier n'y sont pas très différentes.

Dans les 4 groupes d'âges ainsi reconstitués, l'analyse de variance indique évidemment que la hauteur des plus âgés est la plus grande,

Tableau II. Analyse discriminante sur les 8 groupes initiaux de 40 placettes. Distances entre parcelles (métrique de Mahalanobis).

\begin{tabular}{|c|c|c|c|c|c|c|c|c|}
\hline & \multicolumn{8}{|c|}{ Distances entre parcelles } \\
\hline & 36 & 67 & 68 & $110 / 1$ & $110 / 2$ & $113 / 1$ & $113 / 2$ & 117 \\
\hline 36 & 0 & & & & & & & \\
\hline 67 & 1,3327 & 0 & & & & & & \\
\hline 68 & 1,1478 & 0,2438 & 0 & & & & & \\
\hline $110 / 1$ & 1,2211 & 2,2082 & 2,0573 & 0 & & & & \\
\hline $110 / 2$ & 1,2637 & 2,2951 & 2,1343 & 0,1418 & 0 & & & \\
\hline $113 / 1$ & 1,3318 & 2,3147 & 2,2049 & 0,6342 & 0,7177 & 0 & & \\
\hline $113 / 2$ & 1,3624 & 2,3437 & 2,2206 & 0,4128 & 0,4943 & 0,2615 & 0 & \\
\hline 117 & 0,4286 & 1,6025 & 1,4048 & 0,8781 & 0,8956 & 1,1572 & 1,1128 & 0 \\
\hline
\end{tabular}

Ont été regroupées, en raison de leur faible distance (signalée en gras) : 1) les parcelles $110 / 1$ et $110 / 2$; 2) $113 / 1$ et $113 / 2 ; 3) 36$ et 117. 
Tableau III. Surface moyenne de houppier de l'arbre central (en $\left.\mathrm{m}^{2}\right)$ en 1976.

\begin{tabular}{|c|c|c|c|c|c|}
\hline \multirow[b]{2}{*}{$\begin{array}{l}\text { (p) } \\
\text { (a) }\end{array}$} & \multicolumn{2}{|c|}{ Niveau d'éclaircie } & \multicolumn{3}{|c|}{ (p) Parcelle et (a) âge moyen fin 1976} \\
\hline & $\begin{array}{l}113 \\
70 \text { ans }\end{array}$ & $\begin{array}{l}110 \\
58\end{array}$ & $\begin{array}{l}36 \text { et } 117 \\
53\end{array}$ & $\begin{array}{l}67 \text { et } 68 \\
37\end{array}$ & Moyenne \\
\hline 0 & 19,2 & 15,6 & 12,6 & 4,78 & 13,0 \\
\hline 1 & 17,5 & 16,9 & 11,3 & 5,82 & 12,8 \\
\hline 2 & 16,3 & 14,3 & 10,2 & 6,36 & 11,8 \\
\hline 3 & 17,2 & 12,6 & 10,2 & 6,73 & 11,7 \\
\hline 4 & 19,7 & 12,5 & 14,5 & 7,40 & 13,5 \\
\hline Moyenne & 18,0 & 14,4 & 11,8 & 6,22 & 12,6 \\
\hline
\end{tabular}

Tableau IV. Nombre d'arbres éclaircis, par placette de 1 are, par classe d'âge et par niveau d'éclaircie.

\begin{tabular}{|c|c|c|c|c|c|}
\hline \multirow[b]{2}{*}{$\begin{array}{l}\text { Niveau } \\
\text { d'éclaircie }\end{array}$} & \multirow[b]{2}{*}{$\begin{array}{l}\text { âge } \\
\text { en } 1976\end{array}$} & \multicolumn{4}{|c|}{ Eclaircie } \\
\hline & & $\begin{array}{l}n^{\circ} 1 \\
1976\end{array}$ & $\begin{array}{l}n^{\circ} 2 \\
1981\end{array}$ & $\begin{array}{l}n^{\circ} 2 \\
1982\end{array}$ & $\begin{array}{l}n^{\circ} 3 \\
1985\end{array}$ \\
\hline 0 & toutes & \multicolumn{4}{|c|}{ Témoin (éclaircie nulle) } \\
\hline 1 & $\begin{array}{l}37 \\
53 \\
58 \\
70\end{array}$ & $\begin{array}{l}2 \\
1 \\
1 \\
1\end{array}$ & $\begin{array}{l}2 \\
1 \\
0 \\
0\end{array}$ & $\begin{array}{l}0 \\
0 \\
1 \\
1\end{array}$ & $\begin{array}{l}1 \\
0 \\
0 \\
0\end{array}$ \\
\hline 2 & $\begin{array}{l}37 \\
53 \\
58 \\
70\end{array}$ & $\begin{array}{l}4 \\
2 \\
2 \\
2\end{array}$ & $\begin{array}{l}3 \\
2 \\
0 \\
0\end{array}$ & $\begin{array}{l}0 \\
0 \\
2 \\
1\end{array}$ & $\begin{array}{l}1 \\
0 \\
0 \\
0\end{array}$ \\
\hline 3 & $\begin{array}{l}37 \\
53 \\
58 \\
70\end{array}$ & $\begin{array}{l}6 \\
3 \\
3 \\
3\end{array}$ & $\begin{array}{l}4 \\
2 \\
0 \\
0\end{array}$ & $\begin{array}{l}0 \\
0 \\
2 ! \\
1\end{array}$ & $\begin{array}{l}1 \\
0 \\
0 \\
0\end{array}$ \\
\hline 4 & $\begin{array}{l}37 \\
53 \\
58 \\
70\end{array}$ & $\begin{array}{l}30 \\
22 \\
14 \\
9\end{array}$ & $\begin{array}{l}0 \\
0 \\
0 \\
0\end{array}$ & $\begin{array}{l}0 \\
0 \\
0 \\
0\end{array}$ & $\begin{array}{l}0 \\
0 \\
0 \\
0\end{array}$ \\
\hline
\end{tabular}

Pour le niveau d'éclaircie 4, tous les arbres gênant l'arbre de place ont été coupés lors de la première éclaircie. Cela correspond à une mise à l'espacement définitif, dans l'hypothèse d'une densité finale de 100 tiges par hectare.

mais la comparaison faite avec les tables de production montre qu'il n'y a pas de différences de fertilité entre les parcelles. En ce qui concerne les situations initiales, avant la première éclaircie, il n'y a pas de différences significatives entre les groupes d'arbres choisis pour représenter les 5 niveaux d'éclaircie, aussi bien en ce qui concerne la hauteur $h_{76}$ que la surface terrière $g_{76}$ et la surface de projection de houppier $k_{76}$. Le tableau III donne, à titre d'exemple, les mesures des surfaces de houppier en 1976. 
Le dispositif final comprend donc 320 placettes regroupées en 16 répétitions de 4 classes d'âge et 5 intensités d'éclaircie.

\section{Intensité des éclaircies}

Les éclaircies ont été conduites au profit des arbres centraux, en prélevant un nombre variable d'arbres, à partir du plus gênant. Les intensités d'éclaircie ont été codées en 5 niveaux, correspondant à des prélèvements effectifs (en nombre d'arbres) variables suivant la classe d'âge et suivant le passage considéré (1re ou $2^{\theta}$ éclaircie). Le tableau IV résume ces correspondances.

\section{Résultats}

Les résultats qui suivent ont été obtenus par analyse de la variance. Les expressions : "On observe une forte influence" ou "une très forte influence" signifient que les différences sont significatives au seuils habituels de $5 \%$ ou de $1 \%$.

Accroissements absolus en surface terrière et en surface de houppier:

$\lg _{85}=g_{85}-g_{76}$ et $l k_{81}=k_{81}-k_{76}$.
On observe sur les accroissements en surface terrière une très forte influence de l'âge et de l'intensité d'éclaircie; la surface de houppier croit de façon significativement différente selon l'éclaircie, mais pas selon l'âge (voir Tableau V).

Compte tenu de la variabilité importante des dimensions initiales à l'intérieur des groupes de 16 arbres de même âge moyen et ayant subi le même traitement, on a préféré par la suite fournir des résultats sur les accroissements relatifs.

Accroissements relatifs en hauteur :

$$
\operatorname{IR} h_{81}=\frac{h_{81}-h_{76}}{h_{76}}
$$

L'information la plus intéressante, ici, est que, malgré les importantes différences entre les traitements pratiqués et, par suite, entre les accroissements de houppier (multipliés par un facteur 2 entre les placette témoin, niveau 0 et les placettes "mise à distance définitive", niveau 4) l'éclaircie n'a pas d'effet sur la croissance en hauteur. Ce résultat, obtenu pour le court-terme (5 années de croissance), demanderait à être confirmé sur une plus longue période.

Tableau V. Accroissement moyen de la surface de houppier de l'arbre central (en $\mathrm{m}^{2}$ ) entre 1976 et 1981.

\begin{tabular}{clllll}
\hline Niveau d'éclaircie & \multicolumn{6}{l}{ (p) Parcelle et (a) âge moyen fin 1976 } \\
\hline (p) & 113 & 110 & 36 et 117 & 67 et 68 & Moyenne \\
\hline (a) & 70 ans & 58 & 53 & 37 & \\
\hline 0 & 4,52 & 9,05 & 5,00 & 3,38 & 5,49 \\
1 & 8,46 & 9,98 & 7,23 & 6,09 & 7,94 \\
2 & 8,98 & 13,5 & 7,66 & 7,72 & 9,46 \\
3 & 10,4 & 10,0 & 8,42 & 8,20 & 9,27 \\
4 & 13,2 & 13,9 & 12,9 & 10,3 & 12,6 \\
Moyenne & 9,11 & 11,3 & 8,24 & 7,13 & 8,94 \\
\hline
\end{tabular}


Accroissements relatifs de la surface terrière et de la surface de houppier.

$$
\begin{aligned}
& \operatorname{IR} g_{81}=\frac{g_{81}-g_{76}}{g_{76}} \\
& \operatorname{IR} g_{85}=\frac{g_{85}-g_{76}}{g_{76}} \\
& \operatorname{IR} k_{81}=\frac{k_{81}-k_{76}}{k_{76}}
\end{aligned}
$$

Pour ces 3 accroissements, on observe une forte influence à la fois de l'âge et de l'éclaircie, les peuplements les plus jeunes et les éclaircies les plus fortes conduisant à des taux de croissance plus élevés. $\mathrm{Ce}$ résultat était attendu.

On observe également que les taux de croissance en surface de houppier $I R k_{81}$ sont plus élevés que les taux de croissance en surface terrière. Une fois que le houppier a occupé lespace disponible, son taux de croissance devient négli-

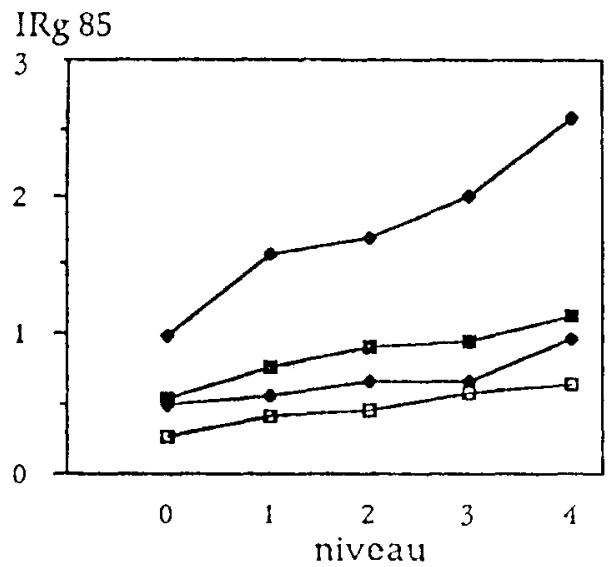

$$
\begin{aligned}
& \rightarrow \text { bloc 4: } 70 \text { ans } \\
& \rightarrow \text { bloc 3: } 58 \text { ans } \\
& - \text { bloc 2: } 53 \text { ans } \\
& \rightarrow \text { bloc 1: } 37 \text { ans }
\end{aligned}
$$

Fig. 1. Accroissement relatif en surface terrière entre 1976 et 1985 par classe d'áge en fonction du niveau d'éclaircie. geable. On en déduit donc que la réaction du houppier aux éclaircies est plus brutale que le redémarrage de l'accroissement du tronc à $1,30 \mathrm{~m}$. En revanche, une fois que l'occupation de l'espace est faite, l'effet de l'éclaircie se prolonge sur l'accroissement du tronc, alors qu'il disparaît sur le développement du houppier.

Influence de l'éclaircie sur les rythmes annuels de croissance.

Remarque préliminaire : ces observations ont été faites lors d'une seule saison de végétation et n'ont donc pas de caractère de généralité.

On n'observe pas d'influence de l'éclaircie sur la date de démarrage de la végétation; en revanche, la date à laquelle $95 \%$ de l'accroissement radial annuel est obtenu passe du 4 septembre pour les éclaircies nulles (niveau 0) au 15 septembre pour les éclaircies fortes (niveau 4). Tout se passe comme si on avait un allongement de la saison de végétation. Cela pourrait s'explic|uer par la compétition pour l'eau en fin d'été, qui est d'autant plus forte que les éclaircies sont plus faibles.

\section{Etudes complémentaires}

L'intensité d'éclaircie ne semble, pour le moment, avoir aucune influence sur les risques de chablis, l'apparition ou la disparition de tares comme le chancre à $\mathrm{NeC}$ tria Ditissima ou les attaques de Cryptococcus fagi. En revanche, les éclaircies semblent provoquer, notamment dans le jeune âge, l'apparition de gourmands et de descentes de cimes chez les arbres peu vigoureux fortement mis en lumière; ce résultat, prévisible d'ailleurs, serait à confirmer. 
Enfin, dans les 2 parcelles les plus jeunes, on a observé des pertes de dominance sur certains arbres de place, et ceci quelle que soit l'intensité des éclaircies.

\section{Discussion - Conclusion}

Les premiers résultats livrés par ce dispositif confirment donc certaines idées empiriques concernant la réaction du hêtre à l'éclaircie : jusqu'à un âge avancé, les arbres peuvent répondre très vigoureusement à de fortes éclaircies en occupant rapidement l'espace libéré et ce, semble-til, sans baisse sensible de la croissance en hauteur. L'effet de stimulation exercé sur l'accroissement ligneux est très marqué également, mais moins brutal que sur le développement du houppier. II semble toutefois que des éclaircies fortes et sélectives au profit d'arbres désignés trop précocement puisse présenter des inconvénients (descente de cime, perte de dominance).

Ces résultats appellent quelques réserves en ce qui concerne :

- la longueur de la période d'observation: il resterait, par exemple, à vérifier sur une plus longue période que des arbres soumis à des densités locales très différentes conservent des croissances en hauteur identiques;

- la définition des traitements : les résultats pourraient être affinés par la prise en compte d'un définition plus explicative des éclaircies : le prélèvement d'un même nombre d'arbres peut en effet, suivant la densité locale initiale, créer une ouverture du couvert variable.

- la taille des placettes : il est probable que, dans un tel dispositif, l'isolement de placettes de faible surface au sein d'un peuplement limite l'éclairement des arbres étudiés par effet d'abri latéral; une confrontation avec des résultats obtenus en peuplement serait prudente : un dispositif installé à Darney (88) devrait permettre, grâce à des placettes unitaires plus grandes, de lever cette dernière réserve.

Par rapport aux placettes permanentes de production - 100 ans pour les plus anciennes - (présentées par Parde, 1980), qui ne décrivent qu'un éventail de sylvicultures assez prudentes et ne fournissent qu'une description individuelle sommaire de chaque arbre, un tel dispositif apparaît très complémentaire : il pourrait, en particulier (Dreyfus, 1988), outre les objectifs affichés en introduction,

- fournir des informations sur la répartition des accroissements entre les branches et le tronc et sur des modifications de la forme des arbres (rectitude, verticalité, coefficient de forme), en fonction de l'intensité d'éclaircie;

- permettre d'étudier l'influence des éclaircies sur les conditions microclimatiques à l'intérieur du peuplement et sur la photosynthèse;

- servir de base à l'étude et à la modélisation de la dynamique du couvert d'une futaie feuillue (par l'intermédiaire de cartographies de houppiers, par exemple : voir figure 2).

Ces différents points feront l'objet de publications ultérieures.

\section{Références}

Dagnelie P. (1975) Analyse statistique à plusieurs variables. Presses Agronomiques de Gembloux, Gembloux

Dreyfus P. (1988) Compétition et croissance dans de jeunes peuplement résineux; aspects biologiques et sylvicoles. Thèse, Aix-Marseille III, avril 1988

Ferrand J.C. (1982) Etude des contraintes de croissance; 2. Variabilité en forêt des 

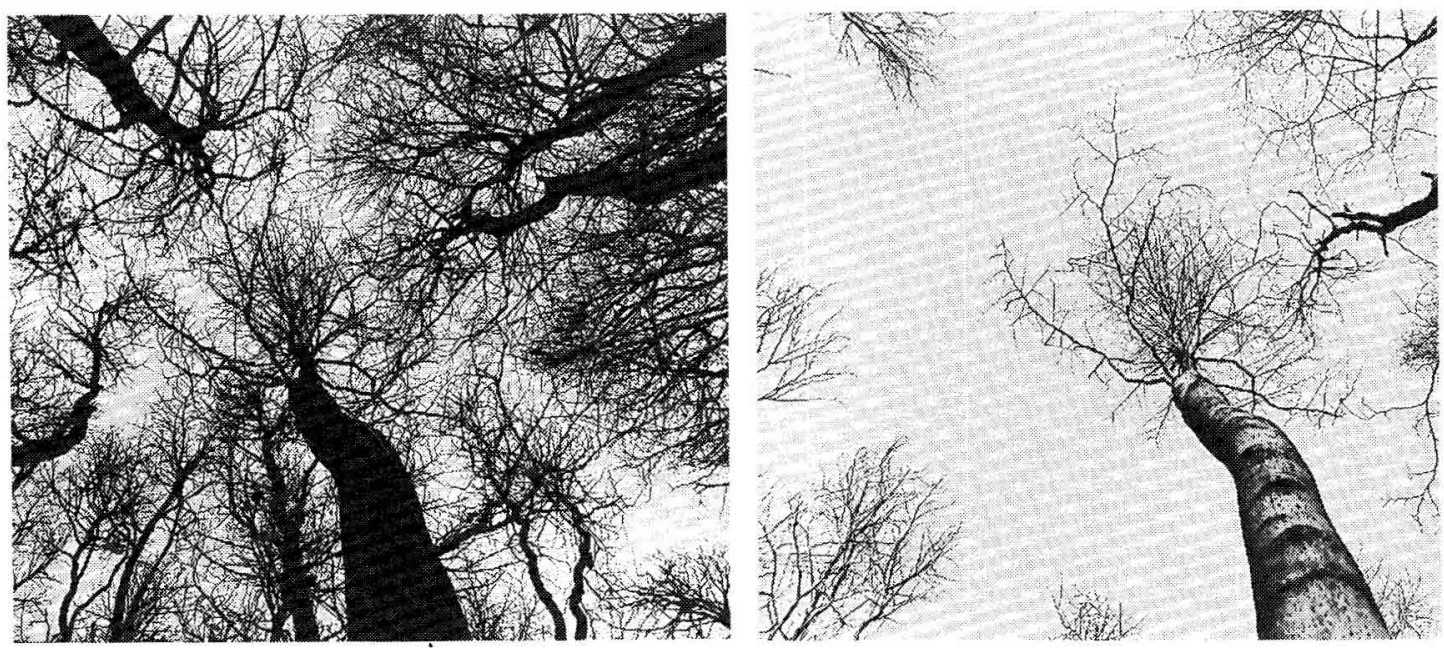

1

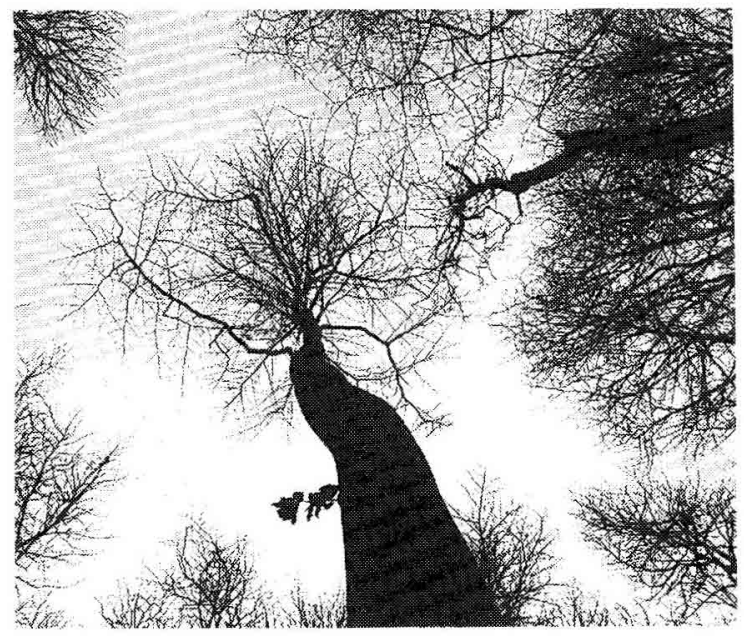

\section{2}
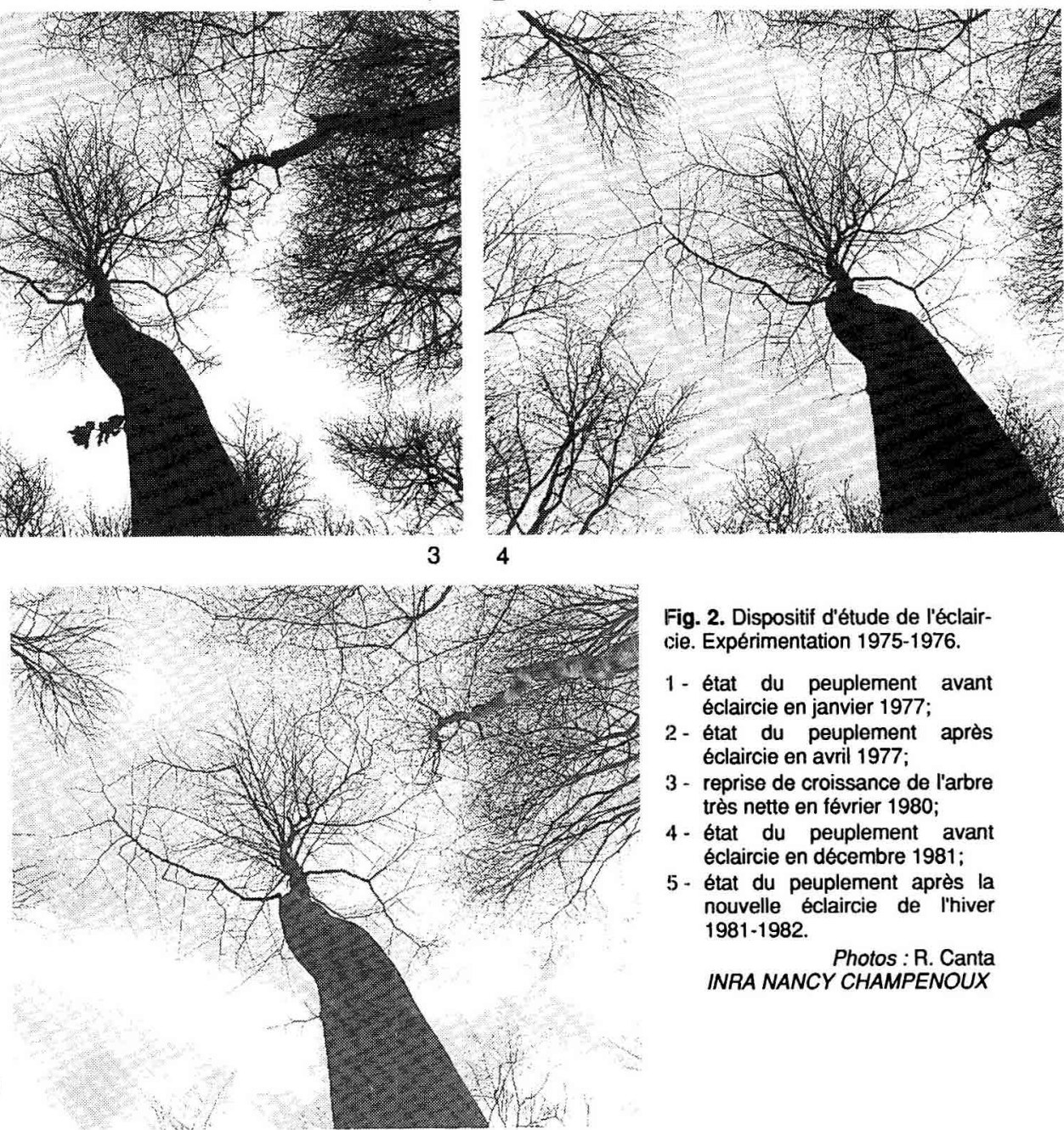

Fig. 2. Dispositif d'étude de l'éclaircie. Expérimentation 1975-1976.

1 - état du peuplement avant éclaircie en janvier 1977;

2- état du peuplement après éclaircie en avril 1977.

3 - reprise de croissance de l'arbre très nette en février 1980;

4- état du peuplement avant éclaircie en décembre 1981;

5- état du peuplement après la nouvelle éclaircie de l'hiver 1981-1982.

Photos : R. Canta INRA NANCY CHAMPENOUX 
contraintes de croissance du hêtre. Ain. Sc. For., 39 (3), 187-217

Keller R., Timbal J. \& Le Tacon F. (1976) La densité du bois de hêtre dans le Nord-Est de la France. Influence des caractéristiques du milieu et du type de sylviculture. Ann. Sci. For., 33 (1), 1-17

Parde J., (1981) De 1882 à 1976/1980 : les places d'expérience de sylviculture du hêtre en forêt domaniale de Haye (Meurthe-et-Moselle). Rev. For. Fr. 33, no sp. 1981, 41-64

Polge H. (1973) Etat actuel des recherches sur la qualité du bois de hêtre Bull. Tech. Off. Nat. For. 73 (4), 13-22

Polge H. (1981) Influence des éclaircies sur les contraintes de croissance du hêtre. Ann. Sci. For. 38 (4), 407-423 